\title{
3D turbulent reconnection driven current-sheet dynamics: solar applications
}

\author{
Lapo Bettarini and Giovanni Lapenta \\ Centrum voor Plasma-Astrofysica, Departement Wiskunde, Katholieke Universiteit Leuven, \\ Celestijnenlaan 200B, 3001 Leuven, Belgium \\ email: lapo.bettarini@wis.kuleuven.be
}

\begin{abstract}
We provide a complete three-dimensional picture of the reconnecting dynamics of a current-sheet. Recently, a two-dimensional non-steady reconnection dynamics has been proved to occur without the presence of any anomalous effect (Lapenta, 2008, Skender \& Lapenta, 2010, Bettarini \& Lapenta, 2010) but such a picture must be confirmed in a full three-dimensional configuration wherein all instability modes are allowed to drive the evolution of the system, i.e. to sustain a reconnection dynamics or to push the system along a different instability path. Here we propose a full-space analysis allowing us to determine the longitudinal and, possibly, the transversal modes driving the different current-sheet disruption regimes, the corresponding characteristic time-scales and to study system's instability space- parameter (plasma beta, Lundquist and Reynolds numbers, system's aspect ratio). The conditions leading to an explosive evolution rather then to a diffusive dynamics as well as the details of the reconnection inflow/outflow regime at the disruption phase are determined. Such system embedded in a solar-like environment and undergoing a non-steady reconnection evolution may determine the formation both of jets and waves influencing the dynamics and energetic of the upper layers and of characteristic down-flows as observed in the low solar atmosphere.
\end{abstract}

Keywords. Instabilities, (magnetohydrodynamics:) MHD, plasmas, Sun: magnetic fields

\section{Introduction}

The power of magnetic fields to affect plasma dynamics on large scales is revealed dramatically by space-borne and ground-base missions (Cirtain et al. 2007, Shibata et al. 2007). The challenge is to capture the dynamics of magnetized plasmas in our simulations, and thus to understand them. Numerical experiments and analytical studies suggested that within the pure resistive MHD framework it is not possible to have a magnetic fieldline reconnecting dynamics that spontaneously evolves from a slow, resistive reconnection regime to a fast, high-power phase. We show how the conversion of magnetic field energy via magnetic reconnection can progress in a fast, fully three-dimensional, volume-filling regime characterized by a chaotic evolution of the system. The process does not require any pre-existing turbulence seed which often is not observed in the host systems prior to the onset of energy conversion. Here, we show the preliminary results for two different magnetic field configurations in a harris-sheet equilibrium (with and without a guide field) which is a well-known system whose (reconnecting) instability dynamics is critical to model plenty of disrupting and explosive phenomena taking place both with the solarterrestrial environment and, in general, in astrophysics.

\section{Numerical settings}

We numerically solve the viscous-(purely) resistive compressible 3D one-fluid MHD equations parametrized by the global Lundquist and kinetic Reynolds number, $S$ and 


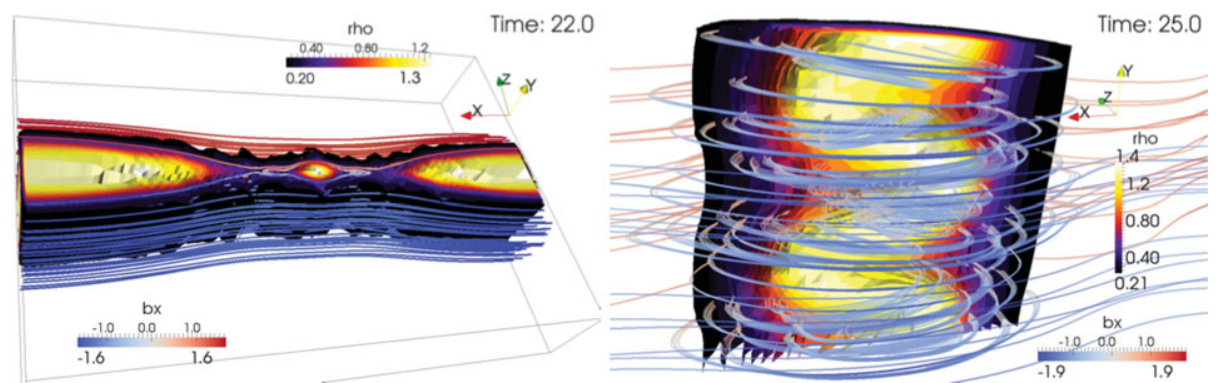

Figure 1. case A. On the right panel, the disruption of the initial laminar current-sheet in a multi-islands configuration (at $t=22$, dimensionless time). Here it is shown the contour of the density and the magnetic field lines whose colors are related to the intensity of the $X$ component of the magnetic field. On the left panel, the disruption in multiple pieces of the current-sheet is followed by a coalescence process (at about $t=25$, dimensionless time) leading to the formation of an unique plasmoid at the centre of the numerical domain box. Afterwards the system is driven by a kinking instability.

$R_{M}$, set to $10^{4}$. We define a stream-wise direction $(X)$, wherein periodic boundary conditions are set at $X=0$ and $L_{X}$, and a cross-stream and span-wise direction ( $Z$ and $Y$ respectively) where we set reflecting boundary conditions. We consider an ideal equation of state with polytropic index $\gamma$ such that $p=\rho(\gamma-1) I$ where $p, \rho$ and $I$ are respectively the kinetic pressure, the density and the enthalpy of the plasma. We use the code FLIP3D-MHD applying a (implicit) particle-in-cell method (FLIP algorithm, (Brackbill, 1991) that allows us (a) to solve an Eulerian-Lagrangian formulation of MHD equations describing the dynamics of our system and (b) to reduce drastically numerical effects. We consider 360 (in $X$ ), 60 (in $Y$ ), 240 (in $Z$ ) Lagrangian markers arrayed initially in a $33 \times 3$ uniform formation in each of the $120 \times 20 \times 80$ cells of our numerical grid. As already pointed out in the introduction, our system consists in a harris-sheet current-sheet with no guide field (case $\mathbf{A}$ ) and with a guide field in the span-wise direction case $\mathbf{B}$ ).

\section{Results}

We provide an exhaustive three-dimensional picture of the reconnecting dynamics of a current-sheet by means of low-diffusive numerical simulations. In both explored cases (A and B) we observe that the initial laminar state spontaneously evolve to a turbulent configuration through a two-stage process. First, the current-sheet is tearing unstable and it breaks to reach a multiple-island configuration (as shown in the left panel of Fig. 1 for case A) undergoing to a coalescence instability that lead to the formation of three-island system with a unique plasmoid at the center of the numerical domain. The presence of the guide field in case B acts to slow down the overall process that anyhow evolves according the same instability path. Secondly, according to the initial configuration we observe a different evolution because the non-steady dynamics critically depends on the interplay of perturbations developing along the magnetic field lines and across them (and so this process is possible only in three-dimensions).

case A: In the right panel of Fig. 1, which is a detailed view of the central plasmoid, it is evident the sinuous mode of the kinking dynamics of the structure and the complex closed loops of the magnetic field following the instability dynamics of the island. This secondary instability destroys the system and drives the high-density fragments which accelerate towards the sides of the box and drive a Rayleigh-Taylor instability of the high-density region there located. 


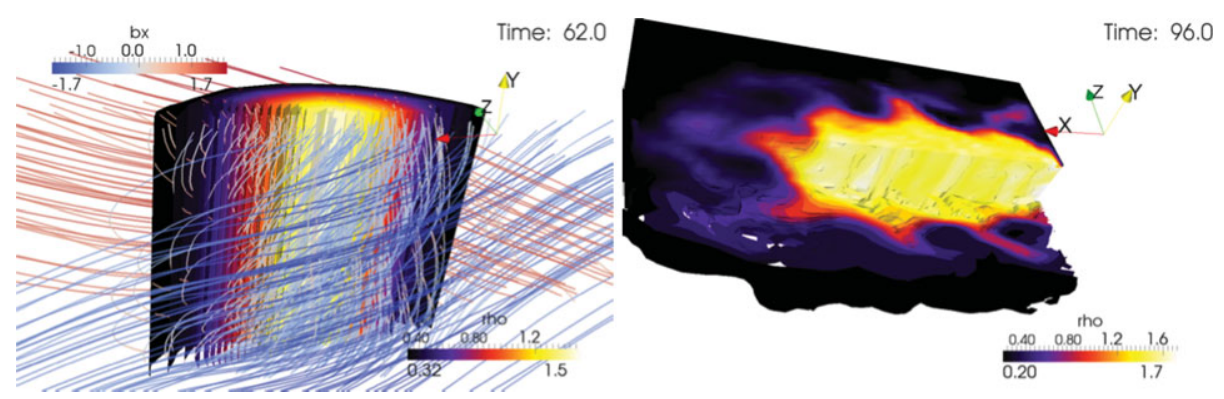

Figure 2. Case B. On the left panel, as in the case A we have a multiple breakdown of the current sheet and the several plasmoids coalesce to form a unique island that is not driven by any three-dimensional instability because of the presence of the guide field: it is shown the contour of the density and the magnetic field lines whose colors are related to the intensity of the $\mathrm{X}$ component of the magnetic field $(t=62$, dimensionless time). On the right panel, clip of the density at the end of the simulation (at about $t=96$, dimensionless time): the disruption of the central island and the related acceleration field trigger a Rayleigh-Taylor instability of the high-density regions located at the sides of the numerical domain.

case B: In the left panel of Fig. 2 the guide field stabilizes the central plasmoid against any kinking evolution and the whole structure is preserved. However the island undergoes the acceleration field resulting from the initial tearing evolution of the current-sheet (and consequent coalescence dynamic): this determines the instability evolution of the highdensity regions at the sides of our box as shown in the right panel of Fig. 2. Here we show that current-sheet system can spontaneously evolve from an initial three- dimensional laminar state to a chaotic configuration via a non-steady self-feeding reconnection dynamics, thus confirming recent previous two-dimensional simulation studies (Lapenta, 2008, Skender \& Lapenta, 2010). Yet the details of the magnetic configuration are key to determine the final state of the instability dynamics. The understanding of mechanisms connecting the micro-scales where reconnection takes place to the evolution of a macroscopic current-sheet configuration is the fundamental step to produce realistic models of all those phenomena requiring fast (and high power) triggering events such solar (stellar) flares and coronal mass ejections (Bettarini \& Lapenta, 2010).

\section{Acknowledgements}

The research leading to these results has received funding from the European Commissions Seventh Framework Programme (FP7/2007 ? 2013) under the grant agreement N. 218816 (SOTERIA project: http://www.soteria-space.eu).

\section{References}

Bettarini, L. \& Lapenta, G. 2010, A\&A, 518, A57

Brackbill, J. U. 1991, J. Comp. Phys., 96, 163

Cirtain, J. W., Golub, L., Lundquist, L., van Ballegooijen, A., Savcheva, A., Shimojo, M., DeLuca, E., Tsuneta, S., Sakao, T., Reeves, K., Weber, M., Kano, R., Narukage, N., \& Shibasaki, K. 2007, Science, 318, 1580

Lapenta, G. 2008, Phys. Rev. Lett., 100, 235001

Shibata, K., Nakamura, T., Matsumoto, T., Otsuji, K., Okamoto, T. J., Nishizuka, N., Kawate, T., Watanabe, H., Nagata, S., UeNo, S., Kitai, R., Nozawa, S., Tsuneta, S., Suematsu, Y., Ichimoto, K., Shimizu, T., Katsukawa, Y., Tarbell, T. D., Berger, T. E., Lites, B. W., Shine, R. A., \& Title, A. M. 2007, Science, 318, 1591

Skender, M. \& Lapenta, G. 2010, Phys. Plasmas, 17, 022905 